\title{
Aplicaciones del SiC biomórfico como reforzante estructural en hormigones refractarios
}

\author{
R. SEPÚLVEDA, M.J. LÓPEZ ROBLEDO, A.R. DE ARELLANO LÓPEZ, J. MARTíNEZ FERNÁNDEZ, C. DOMÍNGUEZ* \\ Dpto. Física de la Materia Condensada, Universidad de Sevilla, 41012 Sevilla, España. * Alfran S.A., Alcalá de Guadaira, Sevilla, España
}

\begin{abstract}
Una posible aplicación del SiC biomórfico (bioSiC) son los reforzante estructural en hormigones refractarios. En este caso se han fabricado piezas de bioSiC con forma de cilindros alargados, 3-4 mm de diámetro y 30-35 mm de longitud, mediante infiltración reactiva de Si líquido en piezas de carbón obtenidas por pirolización de madera de haya de calidad comercial. Hemos estudiado las características microestructurales y las propiedades mecánicas de los reforzantes, como paso previo al estudio de la aplicación mencionada, de la que se ofrece un avance en este trabajo. Para caracterizar la calidad del material y del proceso de fabricación, la microestructura de las piezas se ha estudiado mediante microscopía electrónica de barrido. Los reforzantes de bioSiC fueron ensayados a compresión uniaxial y flexión en cuatro puntos a temperatura ambiente y a alta temperatura $\left(1250-1400^{\circ} \mathrm{C}\right)$ para la determinación de sus propiedades mecánicas, y se realizaron estudios fractográficos en el segundo tipo de ensayos. Subsecuentemente, se prepararon ladrillos refractarios con un $3 \%$ en peso de reforzantes de bioSiC, que fueron curados a diferentes temperaturas (máx. $1600^{\circ} \mathrm{C}$ ). Estos ladrillos se han ensayado en compresión y flexión en tres puntos, a temperatura ambiente, comparándose los resultados con los obtenidos en ladrillos sin reforzantes y reforzados con agujas metálicas de calidad comercial (acero refractario 306 ó 310), con la misma formulación y condiciones de curado.
\end{abstract}

\section{Palabras clave: Biomórfico, SiC, propiedades mecánicas, hormigones refractarios}

\section{Biomorphic SiC: applications as structural reinforcement}

This work is devoted to the study of the time and temperature dependence of the static grain growth in YTZP 4 mol \%, with an average grain size within the submicrometric range $(>0.1 \mu \mathrm{m})$. Also, the mechanical response in the temperature interval between $1200{ }^{\circ} \mathrm{C}$ and $1500{ }^{\circ} \mathrm{C}$ is analysed. The grain growth is controlled by the yttria segregation at the grain boundaries, which plays a key role in the cationic diffusion processes. Microstructural characterization of both as-received and deformed samples allows to conclude that plastic deformation is due to grain boundary sliding (GBS), with stress exponents increasing with the flow stress, but in all cases they are lower than $n=2$.

Keywords: biomorphic, SiC, mechanical properties, microstrucutural characterization, refractory bricks.

\section{INTRODUCCIÓN}

En los últimos años se ha observado un interés creciente en el estudio de cerámicas biomiméticas (1-6). Para el caso del $\mathrm{SiC}$ fabricado a partir de madera (SiC biomófico o bioSiC), las principales ventajas son su bajo coste de producción, la diversidad de microestructuras de las maderas y la posibilidad de generar piezas con formas complejas. Estas características se basan en una estructura de origen biológico, jerarquizada, desarrollada y optimizada por los procesos evolutivos $(7,8)$ para obtener alta resistencia mecánica, baja densidad y alta conectividad entre poros. El SiC es un material de contrastada potencialidad en aplicaciones a elevadas temperaturas, tales como filtros, porta-catalizadores, resistencias eléctricas, reforzantes estructurales $(9,10)$, inclusive se estudian sus posibles aplicaciones en el área médica como implantes óseos (11).

En la industria de refractarios, el $\mathrm{SiC}$ se añade en las formulaciones para aumentar la conductividad térmica (12). A la vez, se emplean fibras y armaduras metálicas para aumentar la resistencia mecánica y al desgaste $(13,14)$. El empleo de piezas de $\mathrm{SiC}$ alargadas podría combinar ventajosamente estos efectos, en particular por el mejor comportamiento del $\mathrm{SiC}$ a alta temperatura, sin embargo las limitaciones en cuanto al coste de producción de las piezas, que por técnicas tradicionales (sinterizado, mecanizado, extrusión,...) no harían viable su aplicación en masa. El bioSiC por el contrario permite la fabricación a bajo coste de las piezas, cuyo proceso puede ser llevado a escala industrial fácilmente. Este trabajo presenta una primera aproximación a las propiedades de los reforzantes de bioSiC, y sus posibles aplicaciones como reforzantes estructurales.

\section{TÉCNICAS EXPERIMENTALES}

\subsection{SiC Biomórfico}

En este trabajo se ha seleccionado madera de haya [Fagus sylbatica L.] de calidad comercial como precursor del bioSiC. En anteriores estudios, se ha establecido la idoneidad de esta madera para la producción de $\mathrm{SiC}$ biomórfico de microestructura reproducible y alta resistencia mecánica $(2,6,15)$.

En las tiendas especializadas se pueden adquirir tubillones estriados de haya de diferentes diámetros para el montaje de estructuras de madera, que son idóneos para el objetivo de este trabajo. En este caso se han seleccionado tubillones de 
$5 \mathrm{~mm}$ de diámetro que se han cortado en piezas de $40 \mathrm{~mm}$ de longitud. El procedimiento de fabricación se inicia con la pirolización de las piezas de haya, para ello se empleo un horno Thermoline Modelo F1100 a $800^{\circ} \mathrm{C}$ en atmósfera de argón con una tasa de calentamiento de $0.5^{\circ} \mathrm{C} / \mathrm{min}$ y $30^{\circ} \mathrm{C} / \mathrm{min}$ para el enfriamiento, el calentamiento lento de las piezas es vital para evitar la formación de grietas o fisuras. La preforma de carbón obtenida es sometida a un proceso de infiltración reactiva con silicio liquido a $1550^{\circ} \mathrm{C}$ durante 30min en atmósfera de vacío, empleando para ello un horno Thermoline Modelo F45500, detalles del proceso se encuentran en la literatura (6) además de estar protegido por una patente (16) . El producto final mantiene la morfología de las piezas de madera iniciales, por lo que obtenemos tubillones estriados de $\mathrm{SiC}$ de 3-4 mm de diámetro por 30-35mm de longitud (fig. 1). La madera original, secada por procedimientos industriales, tiene una densidad aproximada de 0.70 g. $\mathrm{cm}^{-3}$. La densidad promedio de producto final de bioSiC es $2.50 \mathrm{~g}_{\mathrm{cm}} \mathrm{cm}^{-3}$. Para la realización de este estudio se fabricaron alrededor de 1500 piezas de $\mathrm{SiC}$ biomórfico.

La caracterización microestructural fue realizada en un Microscopio Electrónico de Barrido (MEB) marca Philips modelo XL 30, con un equipo de Espectrometría de Rayos X por espectros de energía (EDX) acoplado. La cuantificación del tamaño y distribución de las fases presentes en cada muestra se realizo mediante el Software Image-Pro Plus 4.5.

Los ensayos mecánicos en los reforzantes se realizaron en un equipo MicroTest EM1/50/FR, con un horno de alta temperatura acoplado a su estructura (Grupo de Materiales Biomiméticos y Multifuncionales, Universidad de Sevilla). Los experimentos de compresión axial se realizaron en la dirección longitudinal de los reforzantes, empleando muestras de $6 \mathrm{~mm}$ de alto y $11 \mathrm{~mm}^{2}$ de área. Se ensayaron 5 muestras a cada temperatura $\left(1250,1300,1350\right.$ y $\left.1400^{\circ} \mathrm{C}\right)$ y se emplearon dos velocidades de deformación 5 y $50 \mu \mathrm{m} / \mathrm{min}\left(1.4 \times 10^{-5} \mathrm{~s}^{-1}\right.$ y $1.4 \times 10^{-4} \mathrm{~s}^{-1}$ respectivamente). En general, los ensayos en compresión se detuvieron antes de la rotura de las muestras, una vez alcanzado el máximo valor de la resistencia.

Los ensayos de flexión en 4 puntos se realizaron de acuerdo a la norma ASTM C1211 (17), con temperaturas de ensayos de
$1200,1250,1300$ y $1350^{\circ} \mathrm{C}$. Las superficies de fractura de las muestras tras estos ensayos se inspeccionaron mediante MEB y medios ópticos.

\subsection{Hormigones refractarios}

Las probetas de hormigón fueron fabricadas de acuerdo a especificaciones y composiciones dadas por la empresa colaboradora. El producto final es un ladrillo refractario de alta alúmina de dimensiones 230x55×64 mm aproximadamente. Se fabricaron tres grupos de ladrillos, según el tipo y distribución del reforzante añadido:

- BioSiC en disposición aleatoria, 3\% en peso (12 barras).

- BioSiC en disposición ordenada, 3\% en peso (12 barras).

- Reforzantes estructurales de tipo metálico (agujas metálicas de acero refractario 306 ó 310) 10\% en peso.

Todos los grupos fueron luego curados a 110, $1000 \mathrm{y}$ $1600^{\circ} \mathrm{C}$. También se fabricó, en las mismas condiciones, una serie de ladrillos sin reforzantes con el objeto de comparar su comportamiento.

Los ensayos mecánicos en los ladrillos refractarios se han realizado en los Laboratorios de I+D de Alfran S.A., se realizaron ensayos de flexión en 3 puntos, de acuerdo a la norma ASTM C78-02 (18) y de compresión de acuerdo a la norma ASTM C116-90 (19), ambos a temperatura ambiente. Luego, se inspeccionaron las superficies de fractura mediante microscopia óptica, MEB, empleando también el detector EDS para analizar cualitativamente la composición química en zonas de interés.

\section{RESULTADOS Y DISCUSIÓN}

\subsection{Caracterización del bioSiC}

La figura 2 muestra la imágenes obtenidas mediante MEB de la preforma porosa de carbono y del material final bioSiC. Se observa como la microestructura del producto

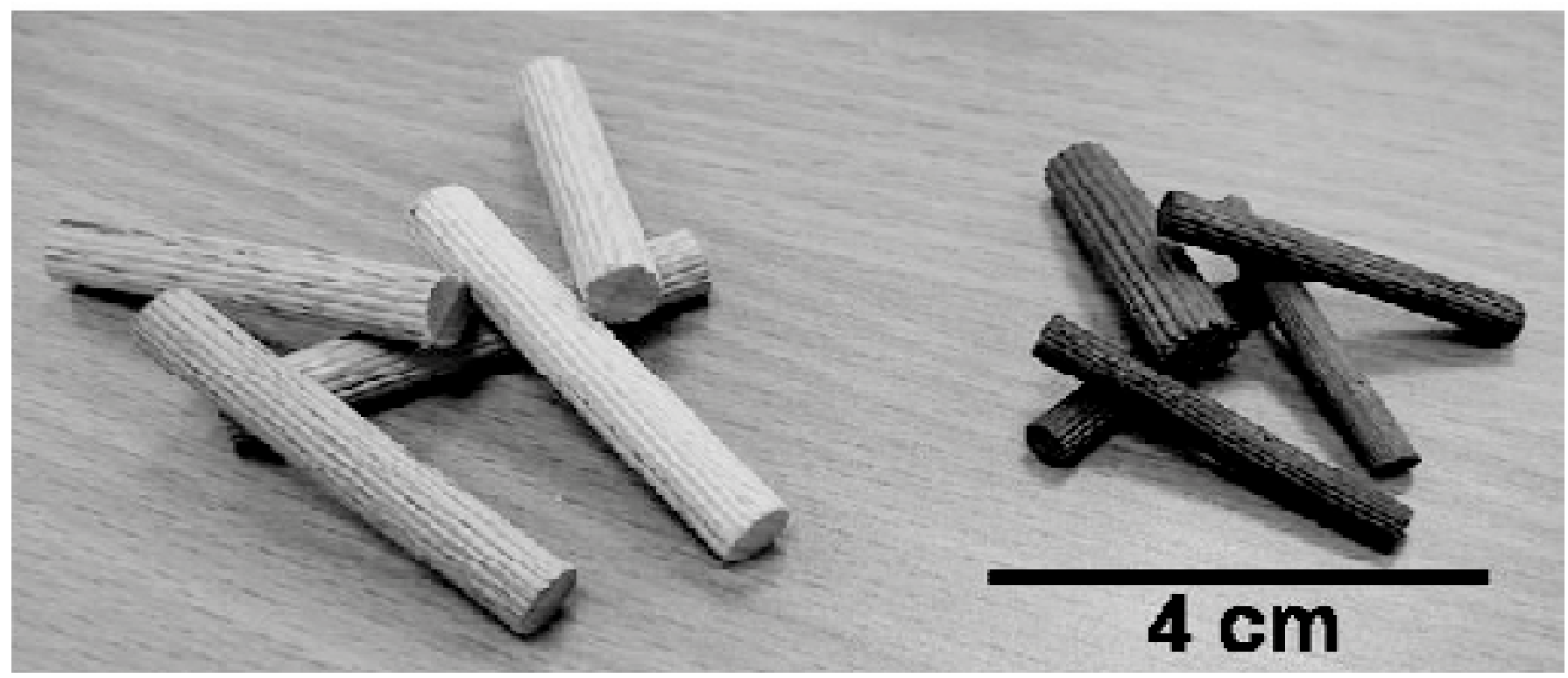

Fig. 1- Reforzantes estructurales de BioSiC con superficie estriada fabricados a partir de madera de haya. 
final mimetiza la de la madera de origen, manteniendo del tamaño de poro en torno a unas $30 \mu \mathrm{m}$, en promedio. Se trata pues, esencialmente, de un compuesto $\mathrm{Si} / \mathrm{SiC}$ en el que las paredes de carbono han reaccionado con el silicio fundido, formando $\mathrm{SiC}$ (tono gris oscuro $\sim 57.8 \%$ del área de la sección transversal), quedando los poros en su mayoría rellenos de $\mathrm{Si}$ sin reaccionar (tono gris claro $\sim 29.2 \%$ del área de la sección transversal). El "esqueleto" de $\mathrm{SiC}$ es pues una estructura altamente interconectada de $\mathrm{SiC}$, cuyo comportamiento parece determinar las propiedades mecánicas del bioSiC $(2,6,20)$. Dos son los defectos más importantes que se observan (tono negro $\sim 13.9 \%$ del área de la sección transversal). Por un lado, algunos poros, de mayor tamaño, quedan vacíos de $\mathrm{Si}$, debido a una menor capilaridad. Por otro, en las zonas en las que los poros son más pequeños, la relación poro/pared de carbono es favorable a lo segundo, quedando pequeñas zonas de $\mathrm{C}$ sin reaccionar debido a una insuficiente velocidad de difusión del Si en el SiC para alimentar el frente de reacción $(21,22)$.
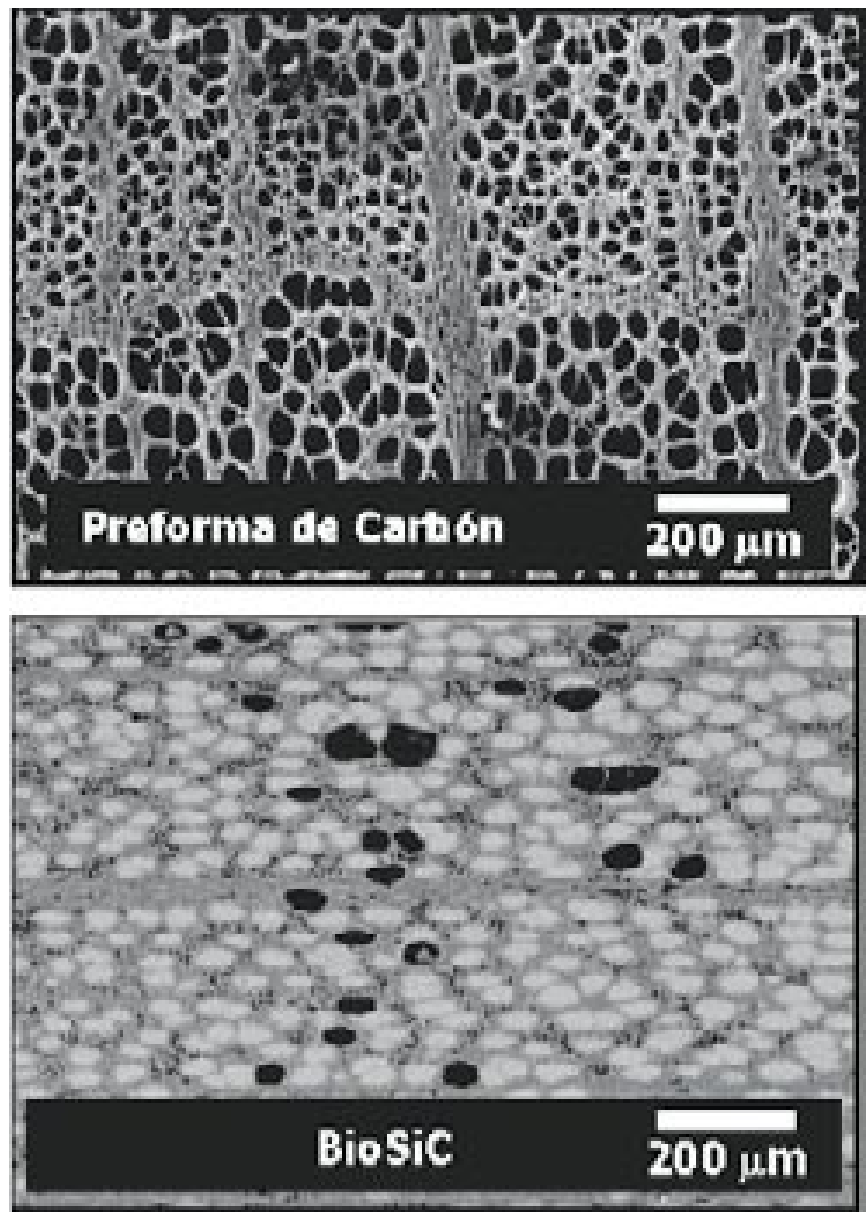

Fig. 2- Fotomicrografías de la preforma de carbono (a) y del BioSiC (b), ambos corte perpendiculares a la dirección axial.

\subsection{Ensayos Mecánicos bioSiC}

La figura 3 presenta el resumen de los resultados de los ensayos mecánicos, de compresión y flexión, realizados sobre los cilindros de bioSiC. En los tipos de ensayos y velocidades de carga empleadas no se observa una pérdida significativa de resistencia hasta superar $\operatorname{los} 1300^{\circ} \mathrm{C}$. Se considera que ello es debido a que la contribución del Si en la estructura del bioSiC, que comienza a disminuir al aproximarse a su temperatura de fusión $\left(1410^{\circ} \mathrm{C}\right)$.

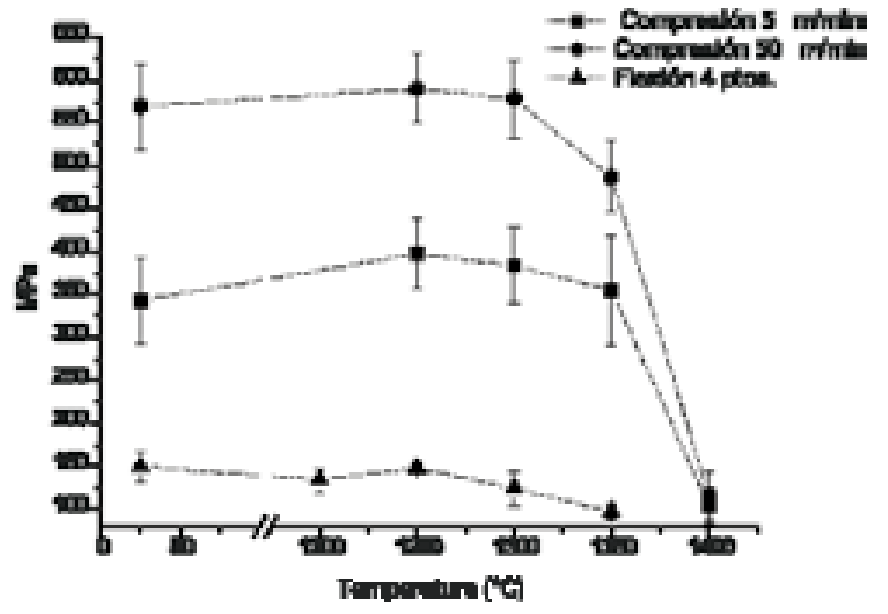

Fig. 3- Resultados de Compresión axial y flexión en 4 puntos.

En los ensayos de compresión se observa también que la resistencia depende de la velocidad de deformación, velocidades de deformación más rápidas dieron lugar a resistencias superiores. Hasta $1300^{\circ} \mathrm{C}$, para la velocidad de deformación más lenta $\left(1.4 \times 10^{-5} \mathrm{~s}^{-1}\right)$, los valores de la resistencia se sitúan entre 350 y $400 \mathrm{MPa}$. En el mismo rango de temperatura, para la velocidad de deformación más rápida $\left(1.4 \times 10^{-4} \mathrm{~s}^{-1}\right)$, la resistencia en compresión asciende a valores entre 550 y $600 \mathrm{MPa}$. La dependencia positiva de la resistencia mecánica con la velocidad de deformación, o lo que es lo mismo, con la velocidad de aplicación de la carga, se ha relacionado típicamente con procesos de rotura dominados por el crecimiento de defectos preexistentes en el material (23), aspecto sobre el que volveremos posteriormente.

Por otra parte, los valores de la resistencia en compresión a altas temperaturas obtenidas en este estudio, sobre piezas fabricadas con una alta variabilidad en la microestructura, y sin mecanizado exterior, resultan comparables a los obtenidos en estudios anteriores sobre bioSiC $(6,24)$ fabricado por procedimientos altamente controlados (16) y de los que se preparaban muestras cuidadosamente mecanizadas. En efecto, los ensayos sobre muestras de haya comercial en la dirección longitudinal, con una velocidad de compresión de $2 \times 10^{-5} \mathrm{~s}^{-1}$, es a $1250^{\circ} \mathrm{C}$ aproximadamente $500 \mathrm{MPa}$ y a $1350^{\circ} \mathrm{C}$ aproximadamente $400 \mathrm{MPa}$ (6), lo que encaja bien con los resultados presentados en la figura 3. El comportamiento a alta temperatura del bioSiC y de otros compuestos $\mathrm{Si} / \mathrm{SiC}$ se ha discutido en detalle en la literatura (20) en base a la teoría de Rice de sólidos porosos en la que las propiedades mecánicas son dominadas por la mínima área sólida $(25,26)$. En este caso, se trataría esencialmente de un sólido poroso (ya que la contribución del Si se considera despreciable a alta temperatura) cuya porosidad está alineada con el eje de carga. En el régimen de alta temperatura, la dependencia positiva de la velocidad de compresión y la resistencia se debe a la contribución de la plasticidad a la deformación: son precisas cargas mayores para mantener regímenes de velocidad de compresión más rápidos (23).

A temperatura ambiente, en las mismas condiciones geométricas y de velocidad de deformación, la resistencia obtenida en estudios anteriores es entre 2 y 4 veces superior a los resultados de este estudio $(24,27)$. Una sencilla aproximación al valor esperado de la resistencia mecánica, aplicando la regla de las mezclas, empleando valores de referencia de $1200 \mathrm{MPa}$ (28) para el Si y $3400 \mathrm{MPa}$ para el SiC, ambos policristalinos 
(29) sugiere que los valores obtenidos en este estudio deben estar especialmente dominados, en ausencia de deformación plástica, por los defectos superficiales e internos de las muestras, debidos a una fabricación menos cuidadosa y a la ausencia de mecanizado. Precisamente, la dependencia de la resistencia con la velocidad de compresión podría estar relacionada con el crecimiento de los defectos hasta un tamaño crítico durante los ensayos mecánicos (30).

Los resultados de flexión en cuatro puntos en todo el intervalo de temperatura de este trabajo pueden compararse con los obtenidos por Singh y Salem (31) para el bioSiC a partir de madera de arce americano (Arce saccharum), de similar densidad en seco que la haya, y también similar densidad final del producto de bioSiC $\left(2.4 \mathrm{~g} \cdot \mathrm{cm}^{-3}\right)$. El material a base de Arce tiene una mayor resistenca a rotura, $344 \mathrm{MPa}$ para temperatura ambiente y $230 \mathrm{MPa}$ para $1350^{\circ} \mathrm{C}$. Esta diferencia en los resultados se debe a que el material de nuestro estudio posee mayores defectos, porosidades, cavidades vacías en silicio y zonas dispersas de carbono sin reaccionar. En el mismo estudio, la caoba americana (Swietenia macrophylla L.) que da lugar a un producto final de bioSiC de 2.0 g. $\mathrm{cm}^{-3}$, y presenta una resistencia a flexión menor que la del arce en todo el rango de temperatura, con valores similares a los del bioSiC de este estudio obtenido de la haya.

Tanto en este estudio como en el descrito por Singh y Salem (31) se encuentra que la resistencia a flexión es muy poco sensible a la temperatura, por lo que se presume que la resistencia este controlada por la rigidez de la matriz de SiC. Así pues, la rotura esta dominada por los defectos presentes en el material, como se observa en la figura 4 .

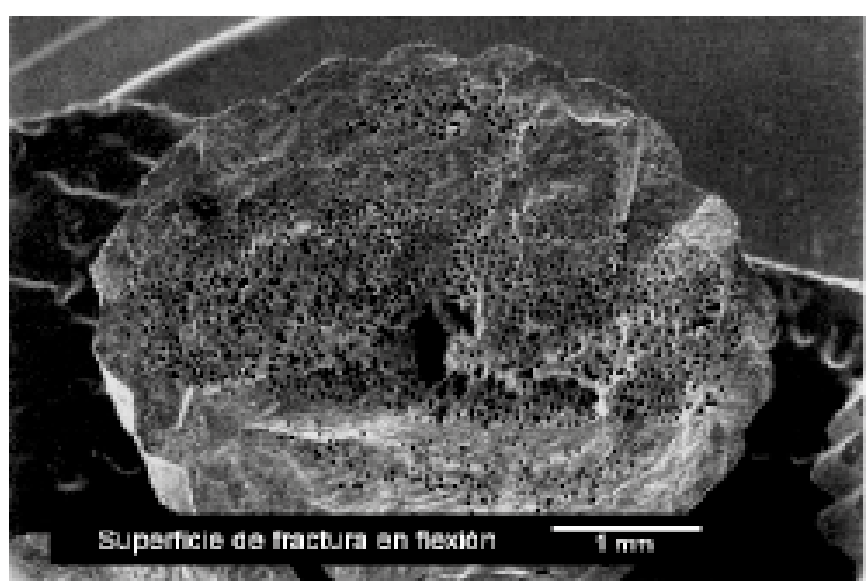

Fig. 4- Superficie de fractura del bioSiC ensayado en flexión a $1250^{\circ} \mathrm{C}$.

A.R. de Arellano y otros (32) han estudiado la evolución de la resistencia a rotura en flexión en cuatro puntos a temperatura ambiente de diversos sistemas $\mathrm{Si} / \mathrm{SiC}$ antes y después de someterlos a ensayos de erosión por choque de partículas de $\mathrm{SiC}$. En el bioSiC a partir del eucalipto blanco (Eucalyptus globulus), y con una densidad de 2.0 g.cm ${ }^{-3}$, la tensión de rotura es $\sim 130 \mathrm{MPa}$, y baja un $25 \%$ tras los ensayos de erosión. El compuesto $\mathrm{Si} / \mathrm{SiC}$ fabricado por reacción $(15 \% \mathrm{Si}+85 \% \mathrm{SiC})$ presenta una tensión de rotura de $\sim 250 \mathrm{MPa}$ (32), que baja un 30\% tras los ensayos de erosión.

De igual forma, los estudios conducidos por Greil P. y otros (15) sobre las propiedades mecánicas de los bioSiC fabricados con diversas maderas. Obtienen valores de $200 \mathrm{MPa}$ en los ensayos de flexión en cuatro puntos para la madera de haya de densidad final $2.58 \mathrm{~g} . \mathrm{cm}^{-3}$ realizados a temperatura ambiente

Se comprueba pues que el comportamiento mecánico de las barras de bioSiC para reforzante estructural, fabricado "en masa", es homologable a otros bioSiC fabricados con un mayor control sobre el procesado.

\subsection{Ensayos en hormigones}

Los ladrillos de hormigón refractario fueron ensayados tanto a compresión como a flexión 3 puntos, los resultados

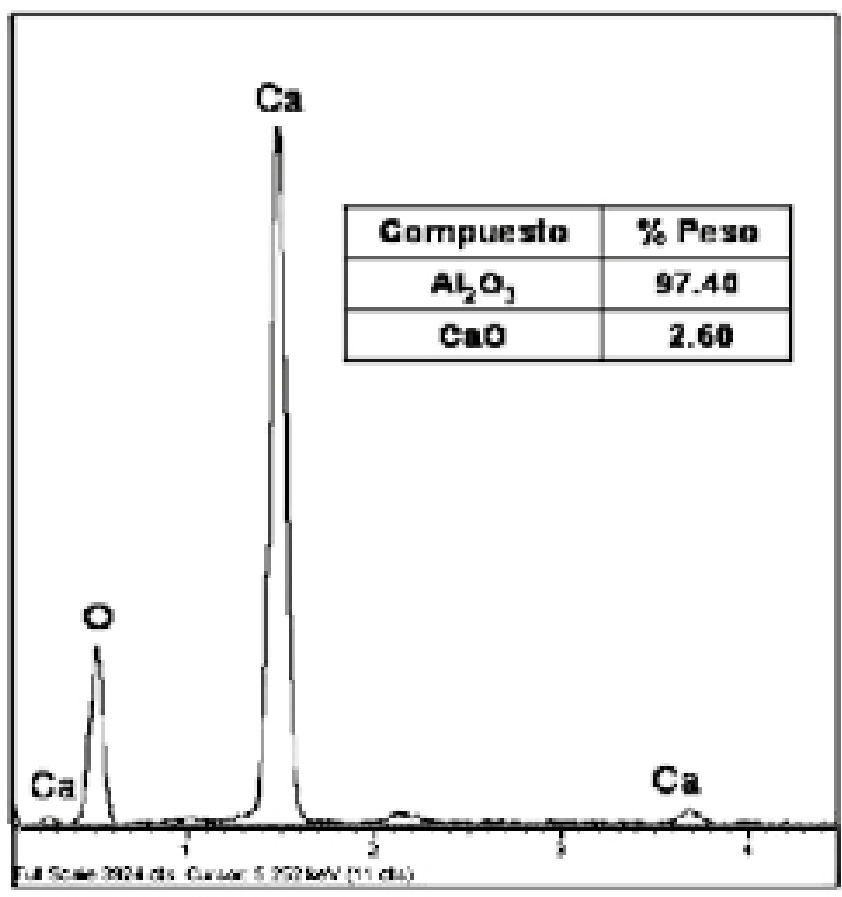

Fig. 5- Espectros de Rayos X por dispersión de energía (EDX) de la interfase bioSiC - homigón para la muestra curada a $1600^{\circ} \mathrm{C}$.

se resumen en la tabla I. Existe buen acuerdo con los valores descritos en otras fuentes bibliográficas para hormigones refractarios de bajo y ultra bajo contenido de cemento

TABLA I. PROPIEDADES MECÁNICAS DE LOS LADRILLOS REFRACTARIOS SEGÚN EL TIPO DE REFORZANTE

\begin{tabular}{|c|c|c|c|c|c|c|c|c|}
\hline \multirow[b]{2}{*}{$\begin{array}{l}\text { Temp. de } \\
\text { Curado }\end{array}$} & \multicolumn{4}{|c|}{ Compresión Axial (MPa) } & \multicolumn{4}{|c|}{ Flexión 3 puntos $(\mathrm{MPa})$} \\
\hline & Blanco & SiC Aleatorio & SiC Orientado & Fibras Metálicas & Blanco & $\mathrm{SiC}$ Aleatorio & SiC Orientado & Fibras Metálicas \\
\hline $110^{\circ} \mathrm{C}$ & 29.81 & 37.74 & 32.252 & 41.03 & 7.46 & 9.42 & 8.70 & 8.73 \\
\hline $1000^{\circ} \mathrm{C}$ & 114.01 & 88.53 & 75.605 & 87.09 & 18.54 & 20.13 & 16.13 & 24.83 \\
\hline $1600^{\circ} \mathrm{C}$ & 119.99 & 120.83 & 125.132 & 101.21 & 27.48 & 27.95 & 27.21 & 25.64 \\
\hline
\end{tabular}


$(14,33,34)$. El efecto más notorio sobre las propiedades de los ladrillos deriva de su proceso de curado. Se observa que la sinterización a temperaturas superiores mejora las propiedades mecánicas, lo que no se consigue de forma intensiva mediante la adición de reforzantes. No hay que olvidar, sin embargo, que la adición de reforzantes tiene como objetivo disminuir la fragilidad de los hormigones, no incrementar la resistencia a rotura (14). Por otro lado, como se ha indicado anteriormente, la adición de $\mathrm{SiC}$ a las formulaciones tiene como finalidad mejorar la conductividad térmica, resistencia a la erosión, y a la escoria.

Es posible pues aportar dos conclusiones preliminares. La primera es que la adición de reforzantes de bioSiC no

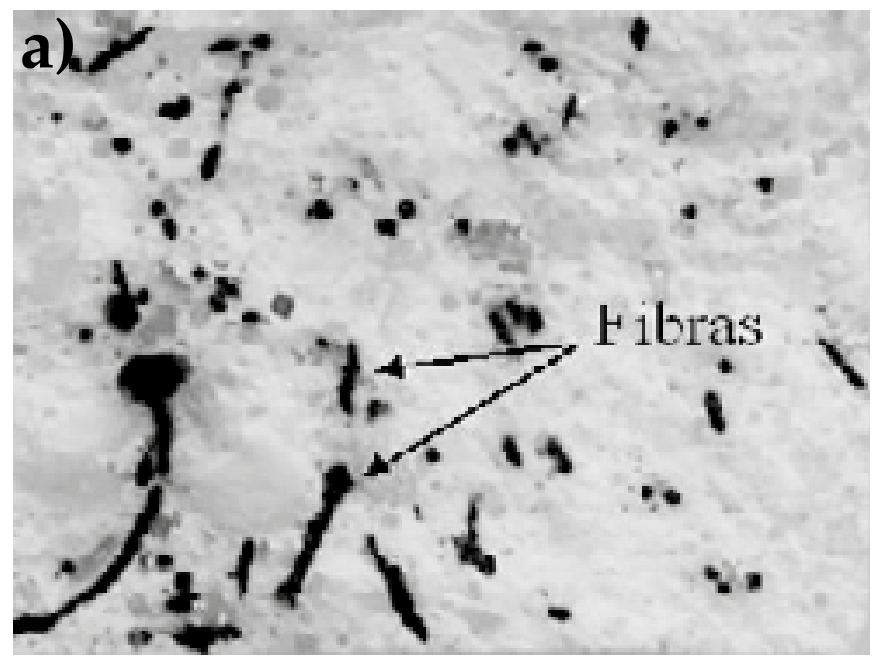

b)

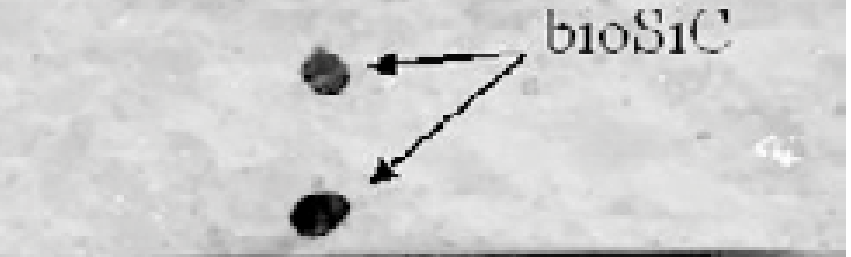

Fig. 6- Hormigones sinterizados a $1600^{\circ} \mathrm{C}$, a) con fibras metálicas y b) con reforzantes de bioSiC.

empeora las propiedades mecánicas. La segunda es que estos reforzantes tienen una mayor estabilidad ante el ciclo térmico de curado, no producen contaminación debida a la oxidación (fig. 5) la cual si se evidencia de forma patente en los ladrillos con reforzantes metálicos curados a $1600^{\circ} \mathrm{C}$ (fig. 6).

Teniendo en cuenta todo esto, parece razonable que futuros desarrollos se dirijan a la producción de piezas más pequeñas en vez de elementos con alta relación de aspecto, ya que las primeras permiten una distribución mas homogénea dentro del ladrillo. Sin embargo esta disminución de tamaño tiene como principal factor limitante la propia microestructura del carbón vegetal.

\section{CONCLUSIONES}

En este estudio se ha comprobado la viabilidad de producir bioSiC a mayor escala, el proceso de fabricación utilizado permite mantener una microestructura aceptable, manteniendo propiedades mecánicas comparables a los bioSiC fabricados mediante procesos altamente controlados. Por lo tanto nuestro proceso de fabricación permite fabricar elementos de bioSiC de una calidad y prestaciones similares a los actuales.

La adición de bioSiC como reforzante estructural en hormigones refractarios produce una respuesta mecánica similar a las formulaciones reforzadas con filamentos metálicos, no que no permite establecer una mejoría en las propiedades estudiadas. Sin embargo, el bioSiC presento una mayor estabilidad química tras el ciclo de curado, contrario a los metálicos que presentaron una mayor oxidación. Esto permite establecer que los reforzantes de bioSiC son térmicamente más estables y no generan una contaminación del ladrillo refractario.

\section{AGRADECIMIENTOS}

Este trabajo se ha realizado con la financiación del Proyecto PETRI PTR 1995-0613-OP. La caracterización microestructural se llevo a cabo en el Centro de Investigación, Tecnología e Innovación CITIUS.

\section{BIBLIOGRAFÍA}

1. T. Ota, M. Takahashi, T. Hibi, M. Ozawa and H. Suzuki, “Biomimetic process for producing SiC wood", J. Am. Ceram. Soc., 78, 12, 3409-3414, 1995

2. Peter Greil, Thomas Lifka and Annette Kaindl, "Biomorphic Cellular Silicon Carbide Ceramics. from Wood: I. Processing and Microstructure", J. Eur. Ceram. Soc., 18, 1961-1973, (1998).

3. Peter Greil, "Near net shape manufacturing if Ceramics.", Mater. Chem. Phys., 61, 64-68, 1999

4. J. Martínez Fernández, F.M. Varela Feria, M. Singh, “High Temperature Compressive Mechanical Behaviour of Biomorphic Silicon Carbide Ceramics.". Scr. Mater., 43, 813-818, 2000

5. J. Martínez Fernández, F. M. Varela Feria y M. Singh, “Microstructure and thermomechanical characterización of biomorphic silicon caride-based Ceramics.", Scr. Mater. 43,813-818 (2001)

6. F. M. Varela Feria, A. R. de Arellano López, J. Martínez Fernández, "Fabricación y propiedades del carburo de silicio biomórfico: maderas cerámicas", Bol. Soc. Esp. Cerm. V., 41, [4] 377-384, 2002.

7. L.J. Gibson, Wood: a natural fibre reinforced composite, Met. Mater., 8, 1992, 333-338

8. Forest Products Laboratory, Wood Handbook-Wood as an Engineering Material, USDA Forrest ervice, Madison, WI, General Technical Reort, FPLGTR-113, 1999

9. Greil P., "Biomorphic Ceramics from lignocellulosics", J. Eur. Ceram. Soc., 21, 2001, 105-118.

10. A. R. de Arellano López, J. Martínez Fernández, P. González, C. Domínguez, V. Fernández-Quero, M. Singh, "Biomorphic SiC: A new engineering ceramic material", Internacional J. of Appl. Ceram. Technol., Vol 1, N $\mathrm{N}^{\mathrm{o}} 1,1-12,2004$

11. P. González, J. Serra, S. Liste, S. Chiussi, B. León, M. Pérez-Amor, J. Martínez Fernández, A. R. de Arellano López, F. M. Valera Feria, "New biomorphic $\mathrm{SiC}$ Ceramics coated with bioactive glass for biomedical applications", Biomaterials, 24, 2003, 4827-4832

12. J.L. Menéndez, "Refractarios en nave de colada de hornos altos", pp. 19-28 en Refractarios Monolíticos, Coord. Carmen Baudín, Ed. Soc. Esp. Ceram. V., 1999

13. Ronald F. Zollo, "Fiber-reinforced Concrete: an overview after 30 years of Development", Cement. Concrete Comp., 19, 107-122, 1997. 
14. Carmen Baudín, "Comportamiento termomecánico de los refractarios monolíticos", pp. 107-128 en Refractarios Monolíticos, Coord. Carmen Baudín, Ed. Soc. Esp. Ceram. V., 1999

15. Peter Greil, Thomas Lifka and Annette Kaindl, "Biomorphic Cellular Silicon Carbide Ceramics from Wood: II. Mechanical Properties", J. Eur. Ceram. Soc., 18, 1975-1983, (1998).

16. Patente: J. Martínez Fernández, F. M. Varela Feria, A. R. de Arellano López, M. Singh. "Method of production of silicon carbide Ceramics from plant precursors" $\mathrm{N}^{\mathrm{o}}$ Solicitud: 02800616.1-2111-ES0200483; Pais: Extensión Internacional de P200102278; Fecha: 04/11/2002; Entidad Titular: Universidad de Sevilla.

17. ASTM C1211, Standard Test Method for Flexural Strength of Advanced Ceramics. at Elevated Temperatures.

18. ASTM C78-02 Standard Test Method for Flexural Strength of Concrete (Using Simple Beam with Third-Point Loading).

19. ASTM C116-90 Test Method for Compressive Strength of Concrete Using Portions of Beams Broken in Flexure (Withdrawn 1999).

20. Julián Martínez Fernández, A. Muñoz, A. R. de Arellano López, F. M. Valera Feria, A. Domínguez-Rodríguez, M. Singh. "Microstructure-mechanical properties correlation in siliconized silicon carbide Ceramics", Acta Mater., 51, 3259-3275, (2003).

1. M. H. Hon, R.F. Davis, "Self-duffision of ${ }^{30} \mathrm{Si}$ in pollcrystalline $\square-\mathrm{SiC}$ ", J. of Mater. Sci., 15, 2073-2080, (1980)

22. M. H. Hon, R.F. Davis, "Self-duffision of ${ }^{14} \mathrm{C}$ in pollcrystalline $\square-\mathrm{SiC}^{\mathrm{C}}$, J. of Mater. Sci., 14, 2411-2421, (1979)

23. J. B. Watchman, "Mechanical Properties of Ceramics", John Wiley and Sons, New Cork, 1996

24. F. M. Varela-Feria, "Fabricación, caracterización microestructural y propiedades mecánicas del carburo de silicio biomórfico", Tesis Doctoral, Universidad de Sevilla, Sevilla 2004

25. Roy W. Rice, Evaluation of the porosity dependence of properties, "Porosity of Ceramics.", 43-99.
26. Thomas H. Courtney, Composite materials, "Mechanical behaviour of materials", 220-262. Michael B. Bever, Charles A. Wert Editors, McGRaw-Hill Publishing Company, 1990

27. Comunicación Privada, Universidad Politécnica de Madrid, Escuela Técnica Superior de Ingenieros de Caminos, Departamento de Ciencias de los Materiales, Madrid, España

28. W. N. Sharpe, Jr., Bin Yuan, R. L. Edwards, “A New Technique for Measuring the Mechanical Properties of Thin Films", J. of Microelectromech. Syst., vol. 6, No 3, September 1997

29. Y. Takeda, K. Nakamura, "Proceeding of the $23^{\text {rd }}$ Japan Congress of Materials Research". Japón (1980) en "Silicon Carbide Ceramics-1. Fundamental and Solid Reaction" editado por S. Somiya y Y. Inomata, Elsevier Applied Science (1991)

30. José Javier Quispe Cancapa, "Propiedades mecánicas de fibras cerámicas cristalinas fabricadas por solidificación direccional", Tesis Doctoral, Universidad de Sevilla, Sevilla 2003

31. M.Singh y J.A. Salem, "Mechanical properties and microstructure of biomorphic silicon carbide Ceramics fabricated from wood precursors", J. Eur. Ceram. Soc., 22 (2002) 2709-2717

32. A.R. de Arellano-López, J. Martínez-Fernández, F.M. Varela-Feria, T.S Orlova, K.C. Goretta, F. Gutierrez-Mora, Nan Chen, J.L. Routbort, "Erosion and strength degradation of biomorphic SiC", J.Eur. Ceram. Soc. 24 (2004) 861-870

33. Santiago Landa, Josefa Molinero, Fernando Carril, "Los revestimientos monolíticos en cucharas desde el punto de vista del fabricante de aceros", pp. 43-53 en Refractarios Monolíticos, Coord. Carmen Baudín, Ed. Soc. Esp. Ceram. V., 1999

34. Especificaciones Técnicas para hormigones de bajo y ultra bajo contenido de cemento, National Refractories and Mineral Corp., Livermore, CA Stephen C. Carniglia, Gordon L. Barna, Handbook of Industrial Refractories Technology, “Design Properties: Mechanical”, 441

Recibido: 09.01 .05

Aceptado: 07.07.05 